\title{
A Study of Near-Field Direct Antenna Modulation Systems Using Convex Optimization
}

\author{
Javad Lavaei, Aydin Babakhani, Ali Hajimiri and John C. Doyle
}

\begin{abstract}
This paper studies the constellation diagram design for a class of communication systems known as near-field direct antenna modulation (NFDAM) systems. The modulation is carried out in a NFDAM system by means of a control unit that switches among a number of pre-designed passive controllers such that each controller generates a desired voltage signal at the far field. To find an optimal number of signals that can be transmitted and demodulated reliably in a NFDAM system, the coverage area of the signal at the far field should be identified. It is shown that this coverage area is a planar convex region in general and simply a circle in the case when no constraints are imposed on the input impedance of the antenna and the voltage received at the far field. A convex optimization method is then proposed to find a polygon that is able to approximate the coverage area of the signal constellation diagram satisfactorily. A similar analysis is provided for the identification of the coverage area of the antenna input impedance, which is beneficial for designing an energy-efficient NFDAM system.
\end{abstract}

\section{INTRODUCTION}

A vast majority of problems in circuits, electromagnetics, and optics can be regarded as the analysis and synthesis of linear systems in the frequency domain. These systems, in the circuit theory, consist of passive elements including resistors, inductors, capacitors, ideal transformers, and ideal gyrators [1]. Since the seminal work [2], there has been remarkable progress in characterizing such passive (dissipative) systems using the concept of positive real functions. This notion plays a vital role not only in circuit design but also in various control problems [1], [3], [4].

The application of control theory in circuit and communication areas evidently goes beyond the passivity concept. Indeed, the emerging optimization tools developed by control theorists, such as linear matrix inequalities (LMIs) [5] and sum-of-squares (SOS) [6], have been successfully applied to a number of fundamental problems in these fields. For instance, the recent paper [7] proposes an LMI optimization to check whether a given multi-port network can be realized using a pre-specified set of linear time-invariant components (namely an inductor and small-signal model of a transistor). Moreover, the work [8] formulates the pattern synthesis of large arrays with bound constraints on the sidelobe and mainlobe levels as a semidefinite program.

It is well-known that a broad class of problems in circuits, electromagnetics, and optics can be formulated as an optimization over the parameters of a multi-port passive network

Javad Lavaei and John C. Doyle are with the Department of Control and Dynamical Systems, California Institute of Technology (emails: lavaei@cds.caltech.edu,doyle@cds.caltech.edu).

Aydin Babakhani and Ali Hajimiri are with the Department of Electrical Engineering, California Institute of Technology, (emails: aydin@caltech.edu, hajimiri@caltech.edu). which is obtained, for instance, via an electromagnetic (EM) simulation. As an example, it is shown in [9] that a strikingly efficient and practical way to deal with certain complex antenna problems is to extract a circuit model and then search for appropriate values of its parameters. The circuit model proposed in [9] is indeed a simple, general model which could be considered the abstract model of different types of problems. Our recent work [10] studies such circuit problems using the available techniques developed in the control theory, especially the LMI and passivity concepts.

The present paper aims to apply our recent results developed in [10] to the problem of constellation diagram design for a class of communication systems, referred to as near-field direct antenna modulation (NFDAM) systems. The objective is to propose a systematic method to design an energy-efficient NFDAM system that is able to send an optimal number of independent signals which can be demodulated reliably.

The rest of the paper is organized as follows. Some preliminaries on NFDAM systems are provided in Section II and the problem is introduced accordingly. The main results are then presented in Section III. The efficacy of this work is elucidated in a practical example in Section IV. Concluding remarks are drawn in Section V. Finally, a proof is given in the appendix.

\section{PRELIMINARIES AND PROBLEM FORMULATION}

Since the invention of the radio in the end of nineteen century, there have been major revolutions in the architecture of the radio but there has been a central component that all of them had in common. In all of these systems, the information is generated before the antenna and the role of the antenna is only to efficiently transmit the signal. Different schemes are developed to add information to a carrier signal (e.g. a sinusoidal waveform). The act of adding information to a carrier signal, known as modulation, can be achieved by altering some properties of the carrier signal such as its frequency, amplitude, or phase. In a broad range of wireless communication systems, the information is generated in low frequencies (base-band frequency region), and then upconverted to a carrier frequency (RF) via a mixer that acts as a multiplier.

The base-band data forms a series of complex numbers that can be separated into real and imaginary parts. The first set is called the In-phase signal (I) that is the real part of the complex signal and the second set is called the Quadrature-phase signal $(\mathrm{Q})$ that is the imaginary part of the complex signal. A simple constellation diagram can 


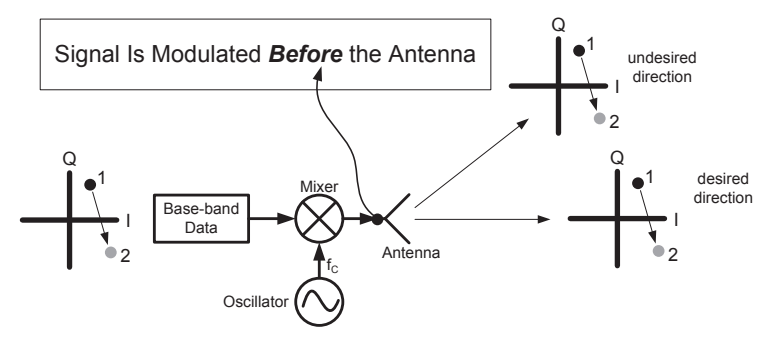

Fig. 1. Modulation before the antenna

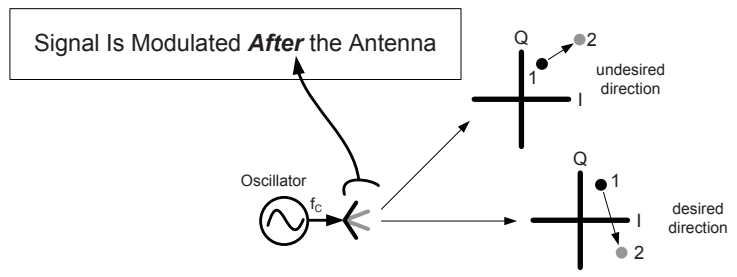

Fig. 2. Modulation after the antenna [9].

be used to represent this complex signal, where each point of the diagram corresponds to some information symbol. After modulating an incoming signal, a conventional antenna propagates the modulated signal in almost all directions. This signal can ideally be received in different directions after some time delay and power attenuation. This implies that the signal cannot be transmitted securely only in some desired directions, and indeed the underlying transmission technique does not differentiate between desired and undesired directions. This fact is illustrated in Figure 1.

A method is proposed in the recent papers [9] and [11] for secure wireless transmission, which allows for generating information by varying the characteristics of the antenna itself as opposed to modulating a signal before the antenna. This idea is illustrated in Figure 2, and will be further elaborated in the next section. This has made it possible to simultaneously transmit independent information to different directions in space. The feasibility of this idea was proved by implementing a silicon-based transmitter leading to the chip given in Figure 3. An unmodulated carrier signal drives an on-chip dipole antenna in this chip, and 90 switches and 10 reflectors are used to vary the characteristics of the antenna (the near-field boundary conditions around the antenna). Note that antennas with time-varying boundary conditions are able to transmit independent signals to different directions simultaneously. The systems based on this concept are said to be near-field direct antenna modulation (NFDAM) transmitters.

The objective of the current paper is to study a NFDAM system with the aim of finding the set of points that can be generated on the signal constellation diagram. As a secondary goal, it is desired to investigate the variation of the input impedance of the antenna and identify the region that the antenna input impedance belongs to.

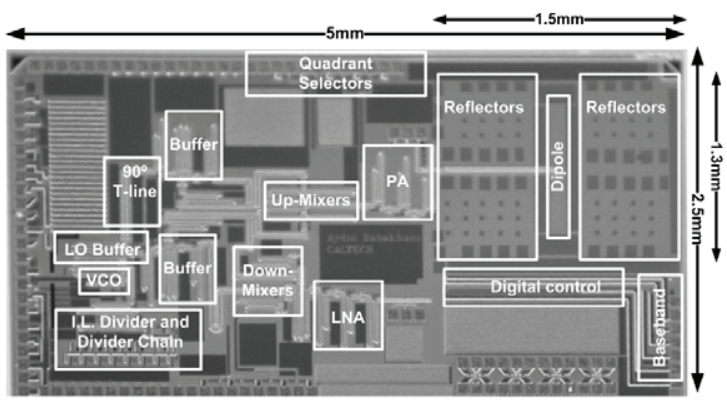

Fig. 3. The first implemented NFDAM transmitter [11].

\section{NEAR-FIELD DIRECT ANTENNA MODULATION}

Consider an antenna configuration consisting of a main transmitting antenna and a number of reflectors where each reflector is accompanied by certain ports (see Figure 6 for an example of this configuration). The objective is to control the ports in such a way that the antenna acts as a secure wireless transmission device that is able to transmit data correctly in one desired direction and scramble data in $z-1$ undesired directions (where $z \in \mathbf{N}$ ). To design a controller for the reflectors' ports, the first step is to place $z$ receiving dipole antennas at the far filed in all desired and undesired directions. Denote with $v_{j}$ the voltage induced on the dipole antenna $j$, for every $j \in\{1,2, \ldots, z\}$. By convention, assume that $v_{1}$ corresponds to the voltage at the desired direction. One can run an electromagnetic simulation at a given frequency $\omega_{0}$ to extract the circuit model of the system. The equivalent circuit model, referred to as Circuit 1, is depicted in Figure 4, which comprises the following ingredients:

- $v_{1}, \ldots, v_{z}$ denote the voltages at the desired and undesired directions in the far field.

- $v_{z+1}, v_{z+2}, \ldots, v_{n}$ denote the voltages on the reflectors' ports in the near field.

- $v_{n+1}$ is the voltage of the input source connected to the transmitting antenna.

- The block "Y-parameter matrix" corresponds to the $Y$ parameter matrix of the antenna system.

- The block "control unit" represents the controller to be designed for the reflectors' ports.

With no loss of generality, assume that $v_{n+1}=1$. Denote the $Y$-parameter matrix of the antenna system at the given frequency $\omega_{0}$ with $Y_{s}$. Moreover, let $z_{\text {in }}$ and $y_{\text {in }}$ represent the input impedance and the input admittance of the antenna system, respectively.

Definition 1: For every arbitrary column vectors $x$ and $y$ of the same dimension, define the norm $\|x+i y\|$ as $\sqrt{x^{*} x+y^{*} y}$ (note that $i$ stands for the imaginary unit).

Given a modulation (complex) point $\alpha$, a problem of interest is how to design the control unit in Circuit 1 so that the received voltage $v_{1}$ at the desired direction becomes equal to $\alpha$ and, in addition, a number of norm constraints 


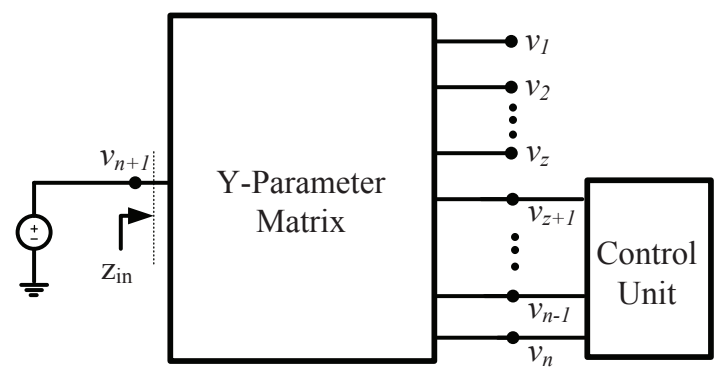

Fig. 4. Circuit 1 modeling the antenna problem under investigation

on $y_{\text {in }}$ and $v_{2}, \ldots, v_{z}$, namely:

$$
\begin{aligned}
\left\|y_{\text {in }}-y_{\text {in }}^{d}\right\| & \leq \varepsilon, \\
\left\|v_{j}-\beta_{j}\right\| & \leq \varepsilon_{j}, \quad j=2,3, \ldots, z
\end{aligned}
$$

are satisfied, where $\beta_{2}, \beta_{3}, \ldots, \beta_{z}$ are given complex numbers and $\varepsilon, \varepsilon_{2}, \ldots, \varepsilon_{z}$ are prescribed tolerances. Note that:

- $y_{\text {in }}^{d}$ is the admittance of the source delivering power to the transmitting antenna. The constraint (1a) is intended to match the input admittance of the transmitting antenna to that of the input source in order to minimize the reflected power.

- For every $j \in\{2,3, \ldots, z\}, \beta_{j}$ is a modulation point, which is sufficiently distant from $\alpha$. The constraint (1b) is imposed to ensure that while the correct symbol $\alpha$ is received at the desired direction, wrong symbols $\beta_{2}, \ldots, \beta_{z}$ are received at the undesired directions.

The simplest type of control unit that one can think of is likely a switching network, which corresponds to Circuit 2 given in Figure 5a. We have shown in our recent work [10] that deciding whether or not there exists a switching control unit that makes the above constraints be satisfied is an NP-complete problem. To alleviate this issue, we have proved that dealing with reciprocal passive control units leads to a convex problem in the form of LMI. Thus, it is henceforth assumed that the control unit being designed is a general reciprocal (linear) passive network (see Circuit 3 in Figure 5b).

An important question arises as to what modulation point $\alpha$ and input impedance $y_{\text {in }}$ can be generated via a passive control unit. Addressing this significant problem is the core of the present work. Define $\mathcal{D}$ to be the set of all modulation points $\alpha$ that can be produced by a passive network in Circuit 3 such that the constraints given in (1) are satisfied. Likewise, define $\mathcal{Q}$ to be the set of all feasible input admittance $y_{\text {in }}$. Note that each of the sets $\mathcal{D}$ and $\mathcal{Q}$ can be identified by a planar region, because every complex number has a 2 -d representation. The sets $\mathcal{D}$ and $\mathcal{Q}$ will be referred to as feasibility constellation region and feasibility admittance region, respectively. It is critical to know the regions $\mathcal{D}$ and $\mathcal{Q}$ before designing an optimal modulation/demodulation scheme. The rest of the paper aims to investigate the shapes of these regions.
Decompose the complex-valued matrix $Y_{s}$ in a block form as:

$$
Y_{s}=\left[\begin{array}{lll}
W_{11} & W_{12} & W_{13} \\
W_{21} & W_{22} & W_{23} \\
W_{31} & W_{32} & W_{33}
\end{array}\right]
$$

where $W_{11} \in \mathbf{C}^{z \times z}, W_{22} \in \mathbf{C}^{(n-z) \times(n-z)}$ and $W_{33} \in \mathbf{C}$ (note that $\mathbf{C}$ denotes the set of complex numbers). Define also $\left\{e_{1}, e_{2}, \ldots, e_{z}\right\}$ to be the set of standard basis vectors of $\Re^{z}$. Throughout this paper, the notation $\succ$ will be used to show inequalities in the positive definite sense. Moreover, the notations $\operatorname{Re}\{\cdot\}$ and $\operatorname{Im}\{\cdot\}$ denote the operators returning the real and imaginary parts of a complex number, respectively.

The following theorem is required for the development of the present work (see our recent paper [10]).

Theorem 1: A point $\alpha$ belongs to $\mathcal{D}$ if and only if there exist symmetric matrices $M, N \in \Re^{(n-z) \times(n-z)}$ and vectors $\tilde{\mathbf{v}}_{1} \in \mathbf{C}^{1 \times z}, \tilde{\mathbf{v}}_{2} \in \mathbf{C}^{1 \times(n-z)}$ such that $\tilde{\mathbf{v}}_{1} e_{1}=\alpha$,

$$
\left[\begin{array}{cc}
\left(\operatorname{Re}\left\{W_{22}-W_{21} W_{11}^{-1} W_{12}\right\}\right)^{-1}-M & N \\
N & M
\end{array}\right] \succ 0,
$$

and:

$$
\begin{aligned}
& \left\|\tilde{\mathbf{v}}_{1} e_{p}-\beta_{p}\right\| \leq \varepsilon_{p}, \quad p=2, \ldots, z \\
& \left\|\tilde{\mathbf{v}}_{1} W_{13}+\tilde{\mathbf{v}}_{2} W_{23}+W_{33}-y_{\text {in }}^{d}\right\| \leq \varepsilon \\
& \tilde{\mathbf{v}}_{1}=-\tilde{\mathbf{v}}_{2} W_{21} W_{11}^{-1}-W_{31} W_{11}^{-1} \\
& \tilde{\mathbf{v}}_{2}=\left(W_{31} W_{11}^{-1} W_{12}-W_{32}\right)(M+N i)
\end{aligned}
$$

If there exist such matrices $M, N$ satisfying the above constraints, then one candidate for the admittance of the passive control unit at the frequency $\omega_{0}$ is:

$$
(M+N i)^{-1}-W_{22}+W_{21} W_{11}^{-1} W_{12} .
$$

Note that if the optimization problem in Theorem 1 is feasible, then $\tilde{\mathbf{v}}_{1}$ and $\tilde{\mathbf{v}}_{2}$ turn out to be equal to the two subvectors of the output voltages, i.e.:

$$
\begin{aligned}
\tilde{\mathbf{v}}_{1} & =\left[\begin{array}{llll}
v_{1} & v_{2} & \cdots & v_{z}
\end{array}\right] \\
\tilde{\mathbf{v}}_{2} & =\left[\begin{array}{llll}
v_{z+1} & v_{z+2} & \cdots & v_{n}
\end{array}\right]
\end{aligned}
$$

It is worth mentioning that the optimization problem proposed in Theorem 1 is indeed an LMI problem. In what follows, an immediate corollary of this theorem will be presented.

Corollary 1: The feasibility constellation and admittance regions $\mathcal{D}$ and $\mathcal{Q}$ are both planar convex sets.

Proof: The proof is a consequence of the fact that the necessary and sufficient conditions provided in Theorem 1 are convex.

That $\mathcal{D}$ is a convex set has an important practical implication: to design an optimal modulation scheme, it is required to identify a maximal set of points in the feasibility constellation region with the minimum point-to-point distance greater than $d$, where $d$ is a given positive number. If $\mathcal{D}$ were a non-convex set with a complicated shape, finding such a maximal set would be a highly complex problem. In contrast, the convexity of the set $\mathcal{D}$ simplifies the design problem significantly, as noted below. 
Define $\mathcal{D}_{u}$ and $\mathcal{Q}_{u}$ to be the feasibility constellation and admittance regions, respectively, in the case when the constraints given in (1) do not exist. In other words, $\mathcal{D}_{u}$ and $\mathcal{Q}_{u}$ are meant to characterize the sets of all possible $v_{1}$ and $y_{\text {in }}$ which could be generated via a passive control unit. Note that $\mathcal{D}$ and $\mathcal{Q}$ are contained in $\mathcal{D}_{u}$ and $\mathcal{Q}_{u}$, respectively, and therefore finding $\mathcal{D}_{u}$ and $\mathcal{Q}_{u}$ leads to understanding how restrictive the imposed constraints are. This is particularly important for a practical design because if the constraints turn out to be too restrictive, the designer may need to relax the constraints to better utilize the system. The following lemma will be later used to study the shapes of $\mathcal{D}_{u}$ and $\mathcal{Q}_{u}$.

Lemma 1: Given a natural number $m$ and real vectors $\mathbf{x}_{1}, \mathbf{x}_{2}, \mathbf{y}_{1}, \mathbf{y}_{2} \in \Re^{m \times 1}$, consider the set of all complex points $\alpha$ for which there exist symmetric matrices $M, N \in \Re^{m \times m}$ such that:

$$
\alpha=\left(\mathbf{x}_{1}^{*}+\mathbf{x}_{2}^{*} i\right)(M+N i)\left(\mathbf{y}_{1}+\mathbf{y}_{2} i\right)
$$

and:

$$
\left[\begin{array}{cc}
-M & N \\
N & M
\end{array}\right] \prec I
$$

This set, denoted by $\mathcal{G}$, is an open circle (ball) centered at the origin with radius:

$$
\sqrt{\left(\left\|x_{1}\right\|^{2}+\left\|x_{2}\right\|^{2}\right)\left(\left\|y_{1}\right\|^{2}+\left\|y_{2}\right\|^{2}\right)}
$$

Proof: The proof is provided in the appendix.

Define:

$$
\begin{aligned}
K_{1}:= & \operatorname{Re}\left\{W_{31} W_{11}^{-1} W_{12}-W_{32}\right\} \\
K_{2}:= & \operatorname{Im}\left\{W_{31} W_{11}^{-1} W_{12}-W_{32}\right\} \\
L_{1}:= & \operatorname{Re}\left\{W_{21} W_{11}^{-1} e_{1}\right\} \\
L_{2}:= & \operatorname{Im}\left\{W_{21} W_{11}^{-1} e_{1}\right\} \\
Q:= & \left(\operatorname{Re}\left\{W_{22}-W_{21} W_{11}^{-1} W_{12}\right\}\right)^{-1} \\
o_{1}:= & -\operatorname{Re}\left\{0.5\left(K_{1}+K_{2} i\right) Q\left(L_{1}+L_{2} i\right)+W_{31} W_{11}^{-1} e_{1}\right\} \\
o_{2}:= & -\operatorname{Im}\left\{0.5\left(K_{1}+K_{2} i\right) Q\left(L_{1}+L_{2} i\right)+W_{31} W_{11}^{-1} e_{1}\right\} \\
r:= & \frac{1}{2}\left(\left\|K_{1} Q^{\frac{1}{2}}\right\|^{2}+\left\|K_{2} Q^{\frac{1}{2}}\right\|^{2}\right)^{\frac{1}{2}} \\
& \times\left(\left\|Q^{\frac{1}{2}} L_{1}\right\|^{2}+\left\|Q^{\frac{1}{2}} L_{2}\right\|^{2}\right)^{\frac{1}{2}} \\
o_{1}^{\prime}:= & -\operatorname{Re}\left\{0.5\left(K_{1}+K_{2} i\right) Q\left(K_{1}^{*}+K_{2}^{*} i\right)\right. \\
& \left.+W_{31} W_{11}^{-1} W_{13}-W_{33}\right\} \\
o_{2}^{\prime}:= & -\operatorname{Im}\left\{0.5\left(K_{1}+K_{2} i\right) Q\left(K_{1}^{*}+K_{2}^{*} i\right)\right. \\
& \left.+W_{31} W_{11}^{-1} W_{13}-W_{33}\right\} \\
r^{\prime}:= & \frac{1}{2}\left(\left\|K_{1} Q^{\frac{1}{2}}\right\|^{2}+\left\|K_{2} Q^{\frac{1}{2}}\right\|^{2}\right)
\end{aligned}
$$

where $Q^{\frac{1}{2}}$ is the unique symmetric positive-definite matrix whose square is equal to the positive-definite matrix $Q$.
Theorem 2: The closure of the convex set $\mathcal{D}_{u}$ is a circle centered at $\left(o_{1}, o_{2}\right)$ with radius $r$. Likewise, the closure of the convex set $\mathcal{Q}_{u}$ is a circle centered at $\left(o_{1}^{\prime}, o_{2}^{\prime}\right)$ with radius $r^{\prime}$.

Proof: It can be inferred from Theorem 1 that a complex point $\alpha$ belongs to $\mathcal{D}_{u}$ if and only if there exist symmetric matrices $M, N \in \Re^{(n-z) \times(n-z)}$ such that:

$$
\left[\begin{array}{cc}
Q-M & N \\
N & M
\end{array}\right] \succ 0
$$

and:

$$
\begin{aligned}
\alpha= & -\left(W_{31} W_{11}^{-1} W_{12}-W_{32}\right)(M+N i) W_{21} W_{11}^{-1} e_{1} \\
& -W_{31} W_{11}^{-1} e_{1}
\end{aligned}
$$

The constraint (11) can be re-arranged as:

$$
\left[\begin{array}{cc}
\tilde{M} & \tilde{N} \\
\tilde{N} & -\tilde{M}
\end{array}\right] \prec I
$$

where:

$$
\begin{aligned}
\tilde{M} & :=2 Q^{-\frac{1}{2}} M Q^{-\frac{1}{2}}-I \\
\tilde{N} & :=2 Q^{-\frac{1}{2}} N Q^{-\frac{1}{2}}
\end{aligned}
$$

The constraint (12) can be expressed in terms of $\tilde{M}$ and $\tilde{N}$ as follows:

$$
\begin{aligned}
\alpha= & -\left(K_{1}+K_{2} i\right)(M+N i)\left(L_{1}+L_{2} i\right)-W_{31} W_{11}^{-1} e_{1} \\
= & -0.5\left(K_{1}+K_{2} i\right)\left(Q^{\frac{1}{2}} \tilde{M} Q^{\frac{1}{2}}+Q+Q^{\frac{1}{2}} \tilde{N} Q^{\frac{1}{2}} i\right) \\
& \times\left(L_{1}+L_{2} i\right)-W_{31} W_{11}^{-1} e_{1} \\
= & -0.5\left(\tilde{K}_{1}+\tilde{K}_{2} i\right)(\tilde{M}+\tilde{N} i)\left(\tilde{L}_{1}+\tilde{L}_{2} i\right)+o_{1}+o_{2} i
\end{aligned}
$$

where:

$$
\tilde{K}_{j}:=K_{j} Q^{\frac{1}{2}}, \quad \tilde{L}_{j}:=Q^{\frac{1}{2}} L_{j}, \quad \forall j \in\{1,2\}
$$

By applying Lemma 1 to the constraints (13) and (15), it can be concluded that $\mathcal{D}_{u}$ is a circle with the aforementioned properties. The proof for the set $\mathcal{Q}_{u}$ can be carried out in the same line, after noting that:

$$
\begin{aligned}
y_{\text {in }} & =-\left(W_{31} W_{11}^{-1} W_{12}-W_{32}\right)(M+N i) \\
& \times\left(W_{21} W_{11}^{-1} W_{13}-W_{23}\right)-W_{31} W_{11}^{-1} W_{13}+W_{33}
\end{aligned}
$$

The details are omitted for brevity.

The first part of Theorem 2 states that the feasibility constellation region $\mathcal{D}_{u}$ is simply a circle with known radius and center. This result significantly benefits the design of an optimal modulation scheme, because finding a maximal set within a circle can be performed systematically. Furthermore, the second part of Theorem 2 says that the feasibility admittance region is again a circle, which is a useful fact for the modulation design. To be more precise, denote the impedance of the input source and the input impedance of the antenna system with $z_{\text {in }}^{d}$ and $z_{\text {in }}$, respectively. The reflection coefficient at the input of the antenna, denoted by $T$, is equal to

$$
T=\frac{z_{\text {in }}-z_{\text {in }}^{d}}{z_{\text {in }}+z_{\text {in }}^{d}}
$$




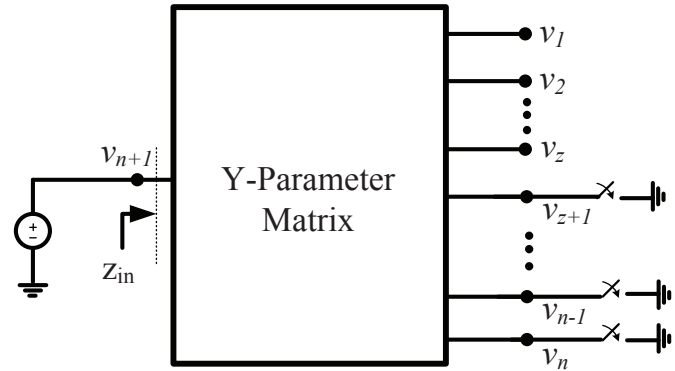

(a)

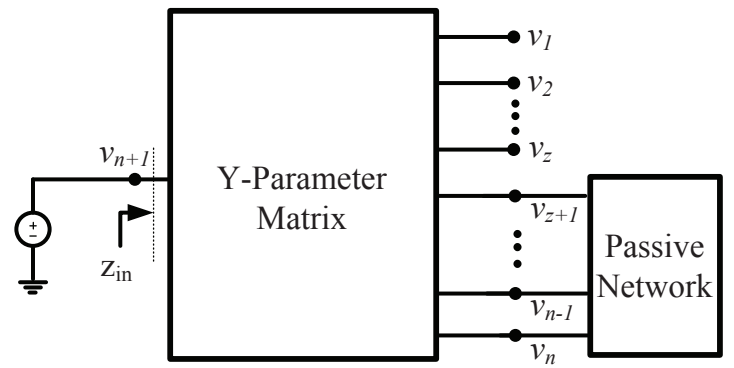

(b)

Fig. 5. (a): Circuit 2 with a switching control unit; (b): Circuit 3 with a passive control unit.

To maximize the power accepted by the antenna, the norm of this reflection coefficient must be minimized. Since the feasibility admittance region is a circle, the feasibility impedance region, denoted by $\tilde{\mathcal{Q}}$, is also a circle whose radius and center can be obtained in terms of $\left(o_{1}^{\prime}, o_{2}^{\prime}\right)$ and $r^{\prime}$. Now, the minimization of the reflected power amounts to finding a point $z_{\text {in }}$ in the circle $\tilde{\mathcal{Q}}$ such that $\|T\|$ is minimum. This problem has a simple analytic solution. In other words, the optimal input impedance that the antenna system accepts by using a passive control unit can be obtained routinely.

After identifying the sets $\mathcal{D}_{u}$ and $\mathcal{Q}_{u}$, and obtaining an optimal input impedance, it is required to find a control unit which makes a number of constraints on the output voltages and the input admittance (i.e. the ones given in (1)) be satisfied. This implies that the modulation scheme must be ultimately designed based on $\mathcal{D}$, rather than $\mathcal{D}_{u}$. It can be seen that even though $\mathcal{D}$ is a convex set, it may not be a circle. The question arises as how to reconstruct the feasibility constellation set $\mathcal{D}$ (or the set $\mathcal{Q}$ ) in the general case. Due to the convexity of the set $\mathcal{D}$ (in light of Corollary 1), a polygon approximation of this set can be found efficiently. Given a positive number $\varepsilon$, consider the problem of finding a simple (non-intersecting) polygon with its vertices on the boundary of $\mathcal{D}$ such that the $x$-distance (i.e. distance in the $x$ direction) between every neighboring vertices of this polygon is less than or equal to $\varepsilon$. Note that as $\varepsilon$ tends to zero, the interior polygon being sought converges to the region $\mathcal{D}$. The following algorithm can be used to determine a polygon satisfying the above-mentioned properties.

\section{Algorithm 1:}

Step 1: Minimize $\operatorname{Re}\left\{v_{1}\right\}$ subject to the constraints (3) and (4). If the optimization problem is feasible, denote the optimal value obtained for $v_{1}$ with $v_{1}^{\min }$. In case of infeasibility of the optimization problem, halt the algorithm because $\mathcal{D}$ is empty.

Step 2: Maximize $\operatorname{Re}\left\{v_{1}\right\}$ subject to the constraints (3) and (4). Denote the optimal value of $v_{1}$ with $v_{1}^{\max }$. Step 3: Set $j=0$.
Step 4: If $j \leq \frac{\operatorname{Re}\left\{v_{1}^{\max }-v_{1}^{\min }\right\}}{\varepsilon}$, minimize $\operatorname{Im}\left\{v_{1}\right\}$ subject to the constraints (3), (4) and $\operatorname{Re}\left\{v_{1}\right\}=\operatorname{Re}\left\{v_{1}^{\min }\right\}+\varepsilon j$, and denote the optimal value of $v_{1}$ with $P_{j}$.

Step 5: If $j \leq \frac{\operatorname{Re}\left\{v_{1}^{\max }-v_{1}^{\min }\right\}}{\varepsilon}$, maximize $\operatorname{Im}\left\{v_{1}\right\}$ subject to the constraints (3), (4) and $\operatorname{Re}\left\{v_{1}\right\}=\operatorname{Re}\left\{v_{1}^{\text {min }}\right\}+\varepsilon j$, and denote the optimal value of $v_{1}$ with $\bar{P}_{j}$.

Step 6: If $j \leq \frac{\operatorname{Re}\left\{v_{1}^{\max }-v_{1}^{\min }\right\}}{\varepsilon}-1$, increment $j$ by 1 and jump to Step 4.

Step 7: The polygon with the vertices $P_{0}, P_{1}, \ldots$, $P_{j}, \bar{P}_{j}, \ldots, \bar{P}_{1}, \bar{P}_{0}$ satisfies the required properties.

The above algorithm obtains an approximating polygon after solving at most $2 \frac{\operatorname{Re}\left\{v_{1}^{\max }-v_{1}^{\min }\right\}}{\varepsilon}+4$ LMIs. The main ideas behind Algorithm 1 are laid out below:

- Steps 1 and 2 are intended to obtain two vertical lines $x=\operatorname{Re}\left\{v_{1}^{\min }\right\}$ and $x=\operatorname{Re}\left\{v_{1}^{\max }\right\}$ tangential to $\mathcal{D}$ between which the region $\mathcal{D}$ is confined.

- Due to the convexity of $\mathcal{D}$, the line $x=\operatorname{Re}\left\{v_{1}^{\min }\right\}+\varepsilon j$, $j \leq \frac{\operatorname{Re}\left\{v_{1}^{\max }-v_{1}^{\min }\right\}}{\varepsilon}$, intersects the region $\mathcal{D}$ in two points $P_{j}$ and $\bar{P}_{j}$. These points are obtained in Steps 4 and 5.

Algorithm 1 presents an efficient optimization-based method to approximate the unknown region $\mathcal{D}$ by a polygon. A derivative of this algorithm can be used for designing an optimal rectangular quadrature amplitude modulation (QAM) scheme. Given a positive constant $d$, consider a constellation diagram $\mathcal{J}$ in the from of rectangular QAM with the points:

$$
\left\{\left(j_{1} d, j_{2} d\right) \mid j_{1} \in\left[-\zeta_{1}, \zeta_{1}\right], j_{2} \in\left[-\zeta_{2}, \zeta_{2}\right], j_{1}, j_{2} \in \mathbf{Z}\right\}
$$

where $\mathbf{Z}$ represents the set of integer numbers. It is desired to obtain the intersection of $\mathcal{D}$ and $\mathcal{J}$, denoted by $\mathcal{Q}_{p}$. Note that $\mathcal{Q}_{p}$ consists of those points in the QAM diagram $\mathcal{J}$ which can be generated by a passive control unit. The following algorithm can be used for this purpose.

\section{Algorithm 2:}

Step 1) Minimize $\operatorname{Re}\left\{v_{1}\right\}$ subject to the constraints (3) and (4). If the optimization problem is feasible, denote the optimal value obtained for $v_{1}$ with $v_{1}^{\min }$. In case 
of infeasibility of the optimization problem, halt the algorithm because $\mathcal{Q}_{p}$ is empty.

Step 2) Maximize $\operatorname{Re}\left\{v_{1}\right\}$ subject to the constraints (3) and (4). Denote the optimal value obtained for $v_{1}$ with $v_{1}^{\max }$.

Step 3) Set $j=0, d_{1}=d\left\lceil\frac{\operatorname{Re}\left\{v_{1}^{\min }\right\}}{d}\right\rceil$ and $d_{2}=$ $d\left\lfloor\frac{\operatorname{Re}\left\{v_{1}^{\max }\right\}}{d}\right\rfloor$ (where $\lceil\cdot\rceil$ and $\lfloor\cdot\rfloor$ are the ceiling and floor operators).

Step 4) If $d_{2}<d_{1}$, the set $\mathcal{Q}_{c}$ is empty and therefore exit the algorithm; otherwise, proceed to the next step. Step 5) If $j \leq \frac{d_{2}-d_{1}}{d}$, minimize $\operatorname{Im}\left\{v_{1}\right\}$ subject to the constraints (3), (4) and $\operatorname{Re}\left\{v_{1}\right\}=d_{1}+j d$, and denote the imaginary part of the optimal value of $v_{1}$ with $y_{j}^{\min }$. Step 6) If $j \leq \frac{d_{2}-d_{1}}{d}$, maximize $\operatorname{Im}\left\{v_{1}\right\}$ subject to the constraints (3), (4) and $\operatorname{Re}\left\{v_{1}\right\}=d_{1}+j d$, and denote the imaginary part of the optimal value of $v_{1}$ with $y_{j}^{\max }$. Step 7) If $j \leq \frac{d_{2}-d_{1}}{d}-1$, increment $j$ by 1 and jump to Step 5.

Step 8) The set $\mathcal{Q}_{p}$ is equal to:

$$
\begin{aligned}
\mathcal{Q}_{p}=\left\{\left(d_{1}+j d, k d\right) \mid\right. & k d \in\left[y_{j}^{\min }, y_{j}^{\max }\right], \\
& \left.j d \in\left[0, d_{2}-d_{1}\right], k, j \in \mathbf{Z}\right\}
\end{aligned}
$$

Note that Algorithm 2 finds the set $\mathcal{Q}_{p}$ after solving at most $4+\frac{d_{2}-d_{1}}{d}$ LMIs. The importance of these algorithms will be further revealed in the simulations provided in the sequel.

\section{Simulation RESUlts}

Consider the antenna configuration depicted in Figure 6, which consists of a transmitting dipole antenna, 10 metal reflectors each with 5 ports (antenna parasitic elements), and a receiving dipole antenna located at the far field in the upward direction. There are 52 ports as follows:

- Port 1 acts as a receiving antenna sampling the radiation pattern of the transmitting antenna at a specific angle in the far field.

- Ports 2 to 51 are intended to change the boundary condition of the transmitting antenna.

- Port 52 corresponds to the transmitting antenna.

The circuit model of the antenna system is extracted at the desired frequency $60 \mathrm{GHz}$ (using localized differential lumped ports) by means of the electromagnetic software IE3D [12]. This model can be either Circuit 2 or Circuit 3, depending on how the impedances of the parasitic elements are designed and implemented. Note that $n$ and $z$ are equal to 51 and 1 , respectively, and that $v_{n+1}=v_{52}=1$.

As the first goal, it is desired to obtain all possible values for $v_{1}$ and $y_{\text {in }}$ in this antenna system under a general passive control unit used to control ports 2 to 51 . Algorithm 1 is deployed to approximate the feasibility constellation and admittance regions $\mathcal{D}_{u}$ and $\mathcal{Q}_{u}$ (with $\varepsilon$ equal to 0.0001). These regions are depicted in Figure 7. It can be seen that both of the regions are circle, which is in accordance with Theorem 2. Due to the circular shape of $\mathcal{D}_{u}$, it is easy to

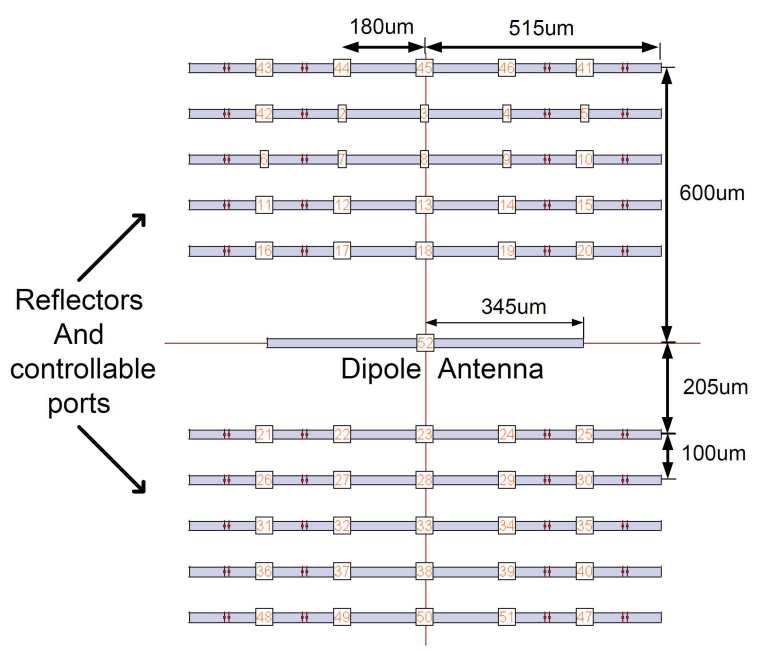

Fig. 6. Configuration of the antenna problem studied in Section IV.

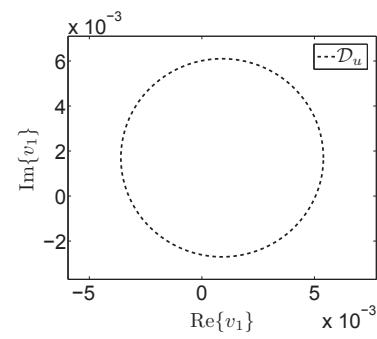

(a)

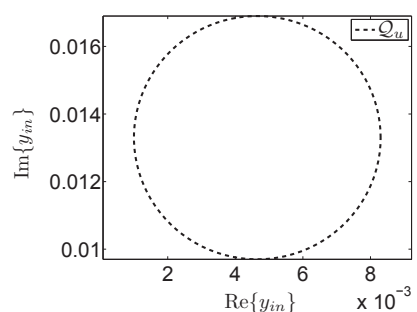

(b)
Fig. 7. (a): The feasibility constellation set $\mathcal{D}_{u}$; (b): the feasibility admittance region $\mathcal{Q}_{u}$.

find an optimal number of modulation points in $\mathcal{D}_{u}$ which are far away from each other by a prescribed number.

To compare the achievable performances of switching and passive control units, denote with $\mathcal{D}_{s}$ the feasibility region for $v_{1}$ under switching control units. Finding the exact shape of $\mathcal{D}_{s}$ requires computing $v_{1}$ for all possible switchings, i.e. $2^{50}$ combinations. Since this may not be possible, a number of switching networks are generated at random and the corresponding values of $v_{1}$ are plotted in Figure 8a. It can be seen that even though a passive network has far more free parameters than a switching network, the region $\mathcal{D}_{u}$ is a fairly good approximation of $\mathcal{D}_{s}$, which can be used for finding the hard limits on the switching performance.

Recall that the feasibility region for $y_{\text {in }}$ is a circle shown in Figure 7b. It is desired to find the set of all possible $v_{1}$ under a passive control unit when the input admittance is matched to the center of this circle. Since $y_{\text {in }}$ is enforced to be fixed, one may speculate that the corresponding feasibility constellation region, denoted by $\mathcal{D}_{1}$, is noticeably smaller than $\mathcal{D}_{u}$. However, it is interesting to note that the set $\mathcal{D}_{1}$ 


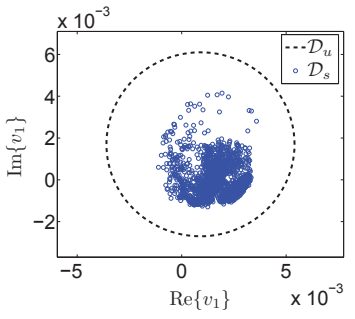

(a)

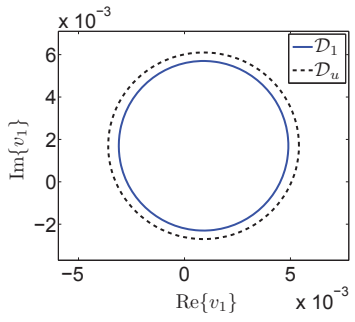

(b)

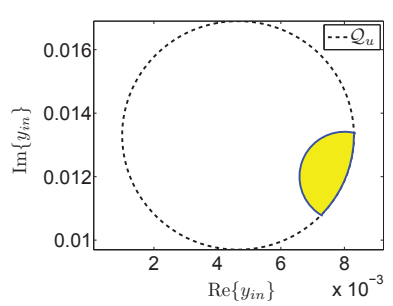

(a)

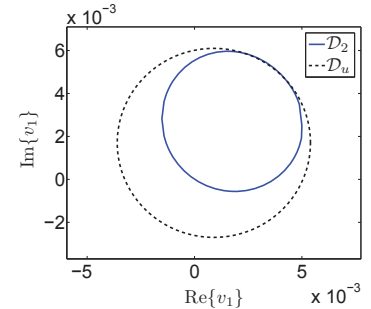

(b)
Fig. 8. (a): The feasibility constellation sets $\mathcal{D}_{s}$ and $\mathcal{D}_{u}$; (b): the feasibility constellation regions $\mathcal{D}_{1}$ and $\mathcal{D}_{u}$.

is only a little smaller than $\mathcal{D}_{u}$, as shown in Figure $8 \mathrm{~b}$. This is due to the high degree of freedom in devising a passive control unit. Notice that $\mathcal{D}_{1}$, found using Algorithm 1 , is a circle despite the fact that imposing a constraint on the input admittance can potentially make the feasibility region non-circular (this will be demonstrated later).

Now, assume that the impedance of the input source is equal to the standard value $50 \Omega$. Since $50 \Omega$ is outside the circular feasibility impedance region, the goal is to find an input impedance (admittance) for the antenna system that minimizes the reflection factor $\|T\|$. By solving a simple geometric problem based on the circle obtained for $\mathcal{Q}_{u}$, the optimal input admittance can be found as $0.008+0.012 i$. Since this number is located on the boundary of $\mathcal{Q}_{u}$, it is expected that imposing $y_{\text {in }}$ to be equal to $0.008+0.012 i$ leads to a feasibility constellation region much smaller than $\mathcal{D}_{u}$. Suppose that the input admittance $y_{\text {in }}$ is permitted to be different from the value $0.008+0.012 i$ by at most $\sqrt{2} \times 10^{-3}$. The corresponding admittance feasibility region is shown in Figure 9a (see the colored area). The feasibility constellation region under this input admittance constraint, denoted by $\mathcal{D}_{2}$, is also plotted in Figure 9b. It can be seen that this region covers a big part of $\mathcal{D}_{u}$ and that $\mathcal{D}_{2}$ is a non-circular (but nearly circular) region.

\section{CONCLusions}

This paper studies the constellation diagram design for a recently introduced communication system that is based on the concept of near-field direct antenna modulation (NFDAM). Unlike the conventional architectures, the signal is modulated in a NFDAM system after the antenna by varying the electromagnetic boundary conditions of the antenna via a passive controller. One of the major challenges in designing an optimal NFDAM system is to find the coverage area of the signal constellation diagram. It is shown that this coverage area is always a convex region that turns into a circle if no constraints are imposed on the parameters of the system. Later on, a linear matrix inequality (LMI) optimization method is proposed to approximate the coverage region by a polygon with any prescribed accuracy. A similar analysis is
Fig. 9. (a): The feasibility admittance constellation (colored area) under the constraint that the input admittance is in a circle centered at $0.008+0.012 i$ with radius $\sqrt{2} \times 10^{-3}$; (b): the feasibility constellation regions $\mathcal{D}_{2}$ and $\mathcal{D}_{u}$.

performed for the identification of the coverage area of the antenna input admittance.

\section{ACKNOWLEDGMENT}

This research was supported by ONR MURI N00014-081-0747 "Scalable, Data-driven, and Provably-correct Analysis of Networks," ARO MURI W911NF-08-1-0233 “Tools for the Analysis and Design of Complex Multi-Scale Networks," and the Army's W911NF-09-D-0001 Institute for Collaborative Biotechnology.

\section{REFERENCES}

[1] N. Nagai, "Linear circuits, systems, and signal processing: advanced theory and applications," Marcel Dekker, 1990.

[2] O. Brune, "Synthesis of a finite two terminal network whose drivingpoint impedance is a prescribed function of frequency," Journal of Mathematics and Physics, vol. 10, pp. 191-236, 1931.

[3] K. S. Narendra and A. M. Annaswamy, "Stable adaptive systems," Dover, 2005.

[4] J. Bao and P. L. Lee, "Process control: the passive systems approach," Springer, 2007.

[5] S. Boyd and L. Vandenberghe, "Convex optimization," Cambridge University Press, 2004.

[6] P. A. Parrilo, "Structured semidefinite programs and semialgebraic geometry methods in robustness and optimization," $P h D$ dissertation, California Institute of Technology, 2000.

[7] J. Harrison, "Formal synthesis of circuits with minimum noise figure using linear matrix inequalities," IEEE Transactions on Circuits and Systems, vol. 54, no. 4, pp. 855-862, 2007.

[8] H. G. Hoang, H. D. Tuan and B. N. Vo, "Low-dimensional SDP formulation for large antenna array synthesis," IEEE Transactions on Antennas and Propagation, vol. 55, no. 6, pp. 1716-1725, 2007.

[9] A. Babakhani, D. B. Rutledge and A. Hajimiri, "Transmitter architectures based on near-field direct antenna modulation," IEEE Journal of Solid-State Circuits, vol. 43, no. 12, pp. 2674-2692, 2008.

[10] J. Lavaei, A. Babakhani, A. Hajimiri and J. C. Doyle, "Solving largescale linear circuit problems via convex optimization," in Proceedings of 48th IEEE Conference on Decision and Control, Shanghai, China, 2009

[11] A. Babakhani, D. B. Rutledge and A. Hajimiri, "Near-field direct antenna modulation, IEEE Microwave Magazine, vol. 10, no. 1, pp. 36-46, 2009.

[12] IE3D electromagnetic simulation and optimization software, Zeland Software Inc., www.zeland.com. 


\section{APPENDIX}

Proof of Lemma 1: The proof will be carried out in two steps. First, the goal is to show that the set $\mathcal{G}$ is contained in the aforementioned circle. To this end, consider a point $\alpha \in$ $\mathcal{G}$ together with its associated matrices $M$ and $N$ satisfying the relations (7) and (8). It can be verified that:

$$
\begin{aligned}
\|\alpha\|^{2} & =\left[\begin{array}{cc}
-\mathbf{x}_{1}^{*} & \mathbf{x}_{2}^{*}
\end{array}\right]\left[\begin{array}{cc}
-M & -N \\
-N & M
\end{array}\right]\left[\begin{array}{cc}
-\mathbf{y}_{1} & \mathbf{y}_{2} \\
\mathbf{y}_{2} & \mathbf{y}_{1}
\end{array}\right] \\
& \times\left[\begin{array}{cc}
-\mathbf{y}_{1}^{*} & \mathbf{y}_{2}^{*} \\
\mathbf{y}_{2}^{*} & \mathbf{y}_{1}^{*}
\end{array}\right]\left[\begin{array}{cc}
-M & -N \\
-N & M
\end{array}\right]\left[\begin{array}{c}
-\mathbf{x}_{1} \\
\mathbf{x}_{2}
\end{array}\right]
\end{aligned}
$$

On the other hand, since the eigenvalues of a Hamiltonian matrix are symmetric, it follows from the inequality (8) that the eigenvalues of the symmetric matrix given in the left side of $(8)$ are all in the interval $(-1,1)$. Hence:

$$
\left[\begin{array}{cc}
-M & -N \\
-N & M
\end{array}\right]^{2} \prec I
$$

Therefore:

$$
\begin{aligned}
& {\left[\begin{array}{cc}
-\mathbf{y}_{1}^{*} & \mathbf{y}_{2}^{*} \\
\mathbf{y}_{2}^{*} & \mathbf{y}_{1}^{*}
\end{array}\right]\left[\begin{array}{cc}
-M & -N \\
-N & -M
\end{array}\right]^{2}\left[\begin{array}{cc}
-\mathbf{y}_{1} & \mathbf{y}_{2} \\
\mathbf{y}_{2} & \mathbf{y}_{1}
\end{array}\right] \prec} \\
& {\left[\begin{array}{cc}
-\mathbf{y}_{1}^{*} & \mathbf{y}_{2}^{*} \\
\mathbf{y}_{2}^{*} & \mathbf{y}_{1}^{*}
\end{array}\right]\left[\begin{array}{cc}
-\mathbf{y}_{1} & \mathbf{y}_{2} \\
\mathbf{y}_{2} & \mathbf{y}_{1}
\end{array}\right]=\left(\left\|\mathbf{y}_{1}\right\|^{2}+\left\|\mathbf{y}_{2}\right\|^{2}\right) I}
\end{aligned}
$$

The above inequality can be manipulated to arrive at:

$$
\begin{aligned}
& {\left[\begin{array}{cc}
-M & -N \\
-N & M
\end{array}\right]\left[\begin{array}{cc}
-\mathbf{y}_{1} & \mathbf{y}_{2} \\
\mathbf{y}_{2} & \mathbf{y}_{1}
\end{array}\right]\left[\begin{array}{cc}
-\mathbf{y}_{1}^{*} & \mathbf{y}_{2}^{*} \\
\mathbf{y}_{2}^{*} & \mathbf{y}_{1}^{*}
\end{array}\right]} \\
& \times\left[\begin{array}{cc}
-M & -N \\
-N & M
\end{array}\right] \prec\left(\left\|\mathbf{y}_{1}\right\|^{2}+\left\|\mathbf{y}_{2}\right\|^{2}\right) I
\end{aligned}
$$

Substituting (24) into (21) leads to:

$$
\begin{aligned}
& \|\alpha\|^{2}<\left[\begin{array}{ll}
-\mathbf{x}_{1}^{*} & \mathbf{x}_{2}^{*}
\end{array}\right]\left(\left\|\mathbf{y}_{1}\right\|^{2}+\left\|\mathbf{y}_{2}\right\|^{2}\right)\left[\begin{array}{c}
-\mathbf{x}_{1} \\
\mathbf{x}_{2}
\end{array}\right] \\
& =\left(\left\|x_{1}\right\|^{2}+\left\|x_{2}\right\|^{2}\right)\left(\left\|y_{1}\right\|^{2}+\left\|y_{2}\right\|^{2}\right)
\end{aligned}
$$

This shows that the point $\alpha$ is inside a circle centered at origin with the radius given by (9).

It remains to show that this circle is contained in $\mathcal{G}$ too. A constructive proof will be provided in the sequel. Let $\alpha$ be an arbitrary point in the underlying circle. Two symmetric matrices $M$ and $N$ satisfying the constraints (7) and (8) are to be constructed. To present the basic idea, assume for now that $x_{2}=y_{2}=0$. Observe that $\operatorname{Re}\{\alpha\}=$ $x_{1}^{*}\left(\frac{\operatorname{Re}\{\alpha\} x_{1} y_{1}^{*}}{\left\|x_{1}\right\|^{2}\left\|y_{1}\right\|^{2}}\right) y_{1}$. This suggests that $M$ be considered as $\frac{x_{1} \operatorname{Re}\{\alpha\} y_{1}^{*}}{\left\|x_{1}\right\|^{2}\left\|y_{1}\right\|^{2}}$. However, the symmetry constraint on $M$ may not be satisfied for this choice of $M$. To resolve this issue, one can symmetrize this term and then define:

$$
M=\operatorname{Re}\{\alpha\} \frac{x_{1} y_{1}^{*}+y_{1} x_{1}^{*}}{\left\|x_{1}\right\|^{2}\left\|y_{1}\right\|^{2}}
$$

It can be seen that the constraint (7) may not hold for this choice of $M$. As a result, the term added in the symmetrization step must be somehow nullified. Define now:

$$
\begin{aligned}
& M:=\operatorname{Re}\{\alpha\} \frac{x_{1} y_{1}^{*}+y_{1} x_{1}^{*}-\left(x_{1}^{*} y_{1}\right) I}{\left\|x_{1}\right\|^{2}\left\|y_{1}\right\|^{2}} \\
& N:=\operatorname{Im}\{\alpha\} \frac{x_{1} y_{1}^{*}+y_{1} x_{1}^{*}-\left(x_{1}^{*} y_{1}\right) I}{\left\|x_{1}\right\|^{2}\left\|y_{1}\right\|^{2}}
\end{aligned}
$$

It is desired to show that the parameters $M$ and $N$ defined above, along with $\alpha$, make the constraints (7) and (8) hold, which in turn proves that $\alpha$ belongs to $\mathcal{G}$. It is easy to observe that (7) is satisfied. To show the validity of (8), it is sufficient to prove that (in light of the inequality $\|\alpha\|<\left\|x_{1}\right\|\left\|y_{1}\right\|$ ):

$$
x_{1} y_{1}^{*}+y_{1} x_{1}^{*}-\left(x_{1}^{*} y_{1}\right) I \leq\left\|x_{1}\right\|\left\|y_{1}\right\| I
$$

Given arbitrary constants $\zeta_{1}, \zeta_{1} \in \Re$, one can write:

$$
\begin{gathered}
\left(\zeta_{1} x_{1}^{*}+\zeta_{2} y_{1}^{*}\right)\left(\left\|x_{1}\right\|\left\|y_{1}\right\| I-x_{1} y_{1}^{*}-y_{1} x_{1}^{*}+\left(x_{1}^{*} y_{1}\right) I\right) \\
\times\left(\zeta_{1} x_{1}+\zeta_{2} y_{1}\right)=\left(\left\|x_{1}\right\|\left\|y_{1}\right\|-x_{1}^{*} y_{1}\right) \\
\times\left(\left\|x_{1}\right\| \zeta_{1}-\left\|y_{1}\right\| \zeta_{2}\right)^{2} \geq 0
\end{gathered}
$$

This shows that the matrix inequality (28) holds (note that the inequality (28) was pre- and post-multiplied by a vector $\zeta_{1} x_{1}^{*}+\zeta_{2} y_{1}^{*}$ rather than a general vector, because the columns of $x_{1} y_{1}^{*}+y_{1} x_{1}^{*}$ are in the span of the vectors $x_{1}$ and $y_{1}$ ).

So far, it is shown how to construct $M$ and $N$ in the case when $x_{2}$ and $y_{2}$ are zero vectors. These matrices can be obtained in the same line for the general case, although the argument is more involved. Note that the staring point for constructing these matrices is to define the matrix $U$ as:

$$
\begin{aligned}
U: & =\left[\begin{array}{cc}
-\mathbf{x}_{1} & \mathbf{x}_{2} \\
\mathbf{x}_{2} & \mathbf{x}_{1}
\end{array}\right]\left[\begin{array}{cc}
-\operatorname{Re}\{\alpha\} & \operatorname{Im}\{\alpha\} \\
\operatorname{Im}\{\alpha\} & \operatorname{Re}\{\alpha\}
\end{array}\right]\left[\begin{array}{cc}
-\mathbf{y}_{1}^{*} & \mathbf{y}_{2}^{*} \\
\mathbf{y}_{2}^{*} & \mathbf{y}_{1}^{*}
\end{array}\right] \\
& \times \frac{1}{\left(\left\|x_{1}\right\|^{2}+\left\|x_{2}\right\|^{2}\right)\left(\left\|y_{1}\right\|^{2}+\left\|y_{2}\right\|^{2}\right)}
\end{aligned}
$$

The main property of $U$ is the following:

$$
\left[\begin{array}{cc}
-\operatorname{Re}\{\alpha\} & \operatorname{Im}\{\alpha\} \\
\operatorname{Im}\{\alpha\} & \operatorname{Re}\{\alpha\}
\end{array}\right]=\left[\begin{array}{cc}
-\mathbf{x}_{1}^{*} & \mathbf{x}_{2}^{*} \\
\mathbf{x}_{2}^{*} & \mathbf{x}_{1}^{*}
\end{array}\right] U\left[\begin{array}{cc}
-\mathbf{y}_{1} & \mathbf{y}_{2} \\
\mathbf{y}_{2} & \mathbf{y}_{1}
\end{array}\right]
$$

If $U$ can be expressed in the form of:

$$
\left[\begin{array}{cc}
-M & -N \\
-N & M
\end{array}\right]
$$

then the corresponding matrices $M$ and $N$ extracted from $U$ satisfy the constraints (7) and (8). This happens when $x_{1}=y_{1}$ and $x_{2}=y_{2}$. Nonetheless, the matrix $U$ must be symmetrized and then the redundant term be compensated in the general case, similar to what was carried out above in the special case $x_{2}=y_{2}=0$. The details are omitted here for brevity. 